\title{
CONOCIMIENTO SOBRE LAS TECNOLOGÍAS DE INFORMACIÓN Y COMUNICACIÓN COMO HERRAMIENTA DE ENSEÑANZA DE LOS DOCENTES UNIVERSITARIOS EN TIEMPOS DE PANDEMIA
}

\author{
Lipselotte de Jesús Infante Rivera ${ }^{1}$ \\ José Manuel Armada Pacheco ${ }^{2}$ \\ Universidad Continental (Perú)
}

Fernando Viterbo Sinche Crispin ${ }^{3}$

Universidad Nacional de Huancavelica (Perú)

Recibido 18/09/2020 Aceptado 30/10/2020

\begin{abstract}
$Z$ La investigación tiene la finalidad analizar el conocimiento sobre el uso de las Tecnologías de la Información y la Comunicación como herramienta de enseñanza de los docentes universitarios en tiempos de pandemia. Dicho estudio se enmarcó dentro de la modalidad de proyecto factible, como un estudio de campo con carácter descriptivo basado en un diseño transeccional y apoyado en un enfoque cuantitativo. En la recolección de los datos primarios y de contenidos se emplearon las técnicas de la revisión documental y la encuesta.
\end{abstract}

5 The research aims to analyze the use of Information and Communication Technologies as a university teaching tool for teachers in times of pandemic. This study was framed within the feasible project modality, as a descriptive field study based on a transectional design and supported by a quantitative approach. In the collection of primary data and content, the techniques of document review and survey were used.

DOI

https://doi.org/10.15366/didacticas2020.23.005

PALABRAs Clave

Tecnologías de la Información y la Comunicación; Herramienta de enseñanza; Pandemia. demic. 


\section{INTRODUCCIÓN}

Las tecnologías de información y comunicación son en la actualidad la herramienta y/o recurso mayormente utilizado por las personas. En el ámbito educativo se ha visto como se ha acrecentado el uso de las tecnologías tanto por parte de los docentes como de los estudiantes. Siendo estos últimos los que mayormente utilizan herramientas tecnológicas para lograr una mayor diversidad en las fuentes de trabajo o actividades educativas. Este es un hecho que se había venido observando desde hace por lo menos una década. Y por lo que ya se sabía que iba en aumento con el avance de dichas tecnologías y la globalización que se encargó de acelerar ese proceso. Sin embargo, pese a que se tenía la certeza de que iba a llegar el momento de utilizar la inteligencia artificial y las muchas herramientas tecnológicas que se ven actualmente, las personas no estaban preparadas para asumir una condición que se puede tornar ilimitada en lo que respecta al uso de la internet como un recurso para hacer sus actividades desde el hogar, llamándolo teletrabajo, trabajo remoto y en otros casos home office.

Este es el caso de la comparación que hace un docente en torno a lo que es la presencialidad con la no presencialidad en su labor educativa, puesto que la mayoría de preferencia desean impartir sus actividades de manera presencial y no a través de la pantalla de un computador. En relación a esto, es importante recordar que no se trata de un trabajo más o de realizar una presentación desde su hogar, sino que en estos momentos ya la virtualidad arropó la mayoría de las formas que eran presenciales en las universidades, haciendo el trabajo solo desde la casa. Y es que, fue una situación que tomó desprevenidos no solo a los docentes sino a los trabajadores a nivel mundial; el hecho de que en marzo de este año 2020 un virus se tornara en pandemia, para dejar a las personas aisladas y no poder salir a realizar sus labores diarias.

En estos casos el dominio las tecnologías de información y comunicación se convierte en un deber que identifica la formación del docente, en donde se puede definir la posibilidad del éxito o el fracaso, como formador educativo. Desde el punto de vista didáctico la introducción de las TIC en las instituciones universitarias provoca necesariamente transformaciones en los procesos de enseñanza y aprendizaje, en las estrategias docentes, en el aprovechamiento del entorno cultural y en los roles de los sujetos o actores educativos.

En este sentido, es importante destacar que el uso de las tecnologías de la información y la comunicación constituyen un objeto de preocupación, debate y reflexión para el entorno educativo universitario. Así pues, uno de los retos más importantes de los profesionales de la educación debe centrarse, sin lugar a dudas, en el uso de estas nuevas tecnologías en el abordaje de las clases virtuales. Estas tecnologías expanden las posibilidades de la comunicación, generan nuevas culturas y posibilitan el desarrollo de nuevas habilidades y formas de construcción del conocimiento; y además es el paliativo perfecto para la situación que se vive por el aislamiento social colectivo. 
Es por esto y por mucho más, que las tecnologías son útiles y además la respuesta ante las necesidades de educarse que tienen los estudiantes; son cada vez más una condición necesaria para la renovación educativa, puesto que el nivel de desarrollo educativo a través de las tecnologías pasa por nuevas herramientas de autodesarrollo de la docencia, gestión educativa, evaluación académica y organización docente, por lo que se hace indispensable formar a los profesores, en el uso y manejo de estas tecnologías para que se adapten a la nueva forma de ser y de trabajar del sector de la enseñanza, es esencial entonces, una apuesta por la formación tecnológica, que conlleve, además, una metodología de apoyo para que el docente pueda evolucionar desde su rol de transmisor de conocimientos a facilitador y guía en la interpretación de los mismos. De esta manera, lo destaca (Urribarrí, 2002):

Con la tecnología podemos crear espacios de aprendizaje de gran riqueza, donde los estudiantes se formen como constructores de conocimiento y nosotros como organizadores de experiencias de aprendizaje... si nosotros creamos espacios para crear conocimiento, los jóvenes podrán compartir lo que están descubriendo cada día y su intercambio cotidiano los llevará a descubrir el valor de la red como tal (p. 5).

Argumentado lo anterior, cabe destacar que hacer uso de las TIC es bastante enriquecedor, puesto que ofrecen la posibilidad de mejorar el proceso enseñanza y aprendizaje, y transformar el quehacer docente alrededor de un nuevo modelo didáctico integrado de trabajo en la red que se desarrolla en un espacio de comunicación o ciberespacio y que cede la iniciativa del proceso formativo al educando que podrá trabajar cooperativamente y acceder a información presentada de manera diferentes (audio, video, texto...). Al mismo tiempo, los educadores adquieren un papel con nuevas prioridades y responsabilidades de mayor complejidad educativa y formadora que, como ocurría en la enseñanza tradicional seguirán marcando el desarrollo de la educación.

Del mismo modo, el hacer uso de las TIC en el aula es un desafío para que los educadores y los estudiantes se preocupen por hacerse más competentes en su uso técnico, tomar conciencia de que no es la cantidad de información que se consigue en la red lo que aporta el conocimiento sino el hecho de que esta sea procesada, analizada, y digerida en la mente, para convertirla en ideas. Con el uso de las TIC se pueden obtener materiales con base en los cuales se puede dar completamente una clase, y al mismo usarlos de mejor manera para obtener ideas que enriquezcan las planificaciones, adaptando las propuestas que se encuentran en la red y rescatando lo que sea útil, minimizando así la tendencia de repetir con nuevas tecnologías lo que siempre se ha hecho sin éstas.

Es evidente, que cada vez la tecnología se implementa con mayor rapidez en más campos laborales, como hay una gran disposición al uso globalizado de estas, la interdisciplinariedad invade la sociedad, ya ningún área queda aislada, todas se interrelacionan y esto es por el uso de la tecnología en el quehacer diario. Los gobiernos buscan la forma de hacer por la vía legal que el uso de la tecnología sea de libre acceso, y la educación es 
un campo que no se puede quedar atrás, es preocupante que en los países con menores recursos los docentes y estudiantes no tienen acceso a esta, mientras que en los países desarrollados esto parece una utopía. En este orden de ideas, (Ávila, 2011 ), enfatiza que:

La integración de las TICs en los procesos educativos se ha convertido en un objetivo prioritario en todos los países desarrollados, debido a entre otras razones, a que las tecnologías digitales, pueden mejorar los procesos de enseñanza a través de la innovación en materiales didácticos, metodologías didácticas más activas y eficaces (p. 93).

Significa desde luego, que es importante que los estudiantes al realizar actividades estén guiados y apoyados por pautas del educador de tal manera que enseñe a explotar la tecnología disponible, sin que para esto sea necesario que la domine, dado que, cuando se trata de integrar las TICs en el aula de clases es muy importante que éstas no se conviertan para los estudiantes tan sólo en una herramienta "entretenida". Es importante mantener claridad en los objetivos planteados antes de comenzar y, en función de estos, promover en los estudiantes el análisis de los recursos y servicios que se les presentan. Y es que, hay que reconocer que en la actualidad el docente se encuentra en desventaja ante el estudiante con el uso y manejo de los dispositivos tecnológicos y la internet como tal. De acuerdo a esto, (Díaz-Barriga, 2013) expresa que

En la actualidad los docentes se encuentran en conflicto ante las nuevas tecnologías, y son varias las razones. Por primera vez en la historia de la humanidad, un alumno está en condiciones de saber más o tener mayor habilidad en el manejo de una computadora que el docente. Los jóvenes de la generación actual, llamados nativos digitales, han crecido y se han desarrollado desde estos instrumentos. No les tienen miedo a las nuevas tecnologías. Diferente situación es para los llamados migrantes digitales, es decir, la mayoría de los docentes, en quienes estos acercamientos a la nueva tecnología pueden resultar complicados, especialmente cuando no encuentran la información que buscan para seguir avanzando en el conocimiento de una nueva tecnología. En este contexto el docente se siente en desventaja con respecto de sus estudiantes (p. 5).

Es por esto, que el docente está llamado a hacer de su actividad docente algo más que proporcionar información que ya conoce y de forma monótona, aburrida y desmotivada. Está en el deber de formarse y/o capacitarse para estar a la par de la nueva generación de los nativos digitales para ir en correspondencia de sus conocimientos, ya que actualmente los jóvenes se encuentran muchas veces mejor preparados que los mismos docentes, ocasionando una pérdida de interés en las clases. En ese sentido es importante que el docente utilice de muy buena forma el internet y las diversas herramientas que ofrece, ha de ser creativo e innovador en ese aspecto, para poder captar la atención del estudiante y mantenerlo concentrado en las clases.

Y es que, la sociedad no ha parado, el avance no se detiene. Se pudo observar en el segundo semestre que va del año como se reactiva día a día la economía, la educación y se 
vuelve a mover todo; pero esta vez con la diferencia que casi todo ha sido de forma digital. El ser humano actual es un ser virtual, es sin lugar a dudas alguien que no se va a quedar con un solo conocimiento y es por ello que sigue a pasos agigantados las nuevas tecnologías. En torno a esto, (Richta, 2010), señala que

el hombre no puede ya conformarse con una serie de conocimientos elementales completados con un aprendizaje artesano-manual, dado que, en base a ellos no le sería posible estar a la altura de los adelantos científico - técnicos ocurridos, ni podría hacerles frente a los cambios que el progreso impone, por lo tanto la carencia de cultura asociada a las aplicaciones tecnológicas informatizadas genera un problema importante para la interpretación de la dinámica social y los avances de la ciencia (p. 26).

De igual manera, la tecnología avanza día a día, y cada vez con mayor velocidad, es notable como lo que hoy representa una innovación, ya mañana es historia. El mundo globalizado en el que se vive no permite lo estático, todo desempeño debe ser dinámico, cambiante y adaptable. Por último, conveniente hacer referencia al papel del docente en este proceso de cambio y desarrollo tecnológico. El docente clásico se proyectaba en sus aulas de manera presencial con una tiza y un borrador, posteriormente empezaron a aparecer algunos otros medios auxiliares de la docencia como pancartas, retroproyectores y proyectores de vistas fijas. Cada uno de estos recursos representaban un reto a la labor del docente, si bien eran considerados medios auxiliares que contribuían a elevar el nivel cualitativo de las clases, no exigían al profesor mucha preparación cognoscitiva de la técnica que se iniciaba como apoyo a la docencia. En palabras de (Velasco, 2009) cuatro son los medios que los profesores consideraban como imprescindibles para la realización de su actividad profesional: la biblioteca, la pizarra, los libros de lecturas personales, y los libros de textos (p. 65).

Como se puede observar no se hace mención a las tecnologías. En unos pocos años esta situación se ha revertido totalmente, ya que actualmente se maneja un lenguaje totalmente diferente, ahora el docente habla sobre las pizarras interactivas, los programas de apoyo para la realización de mapas mentales y conceptuales, en ingeniería se utilizan modelados matemáticos que se expresan a través de un programa computacional, hay calculadoras inteligentes. En fin, existen hoy en día muchas herramientas de apoyo tanto para el docente como para el estudiante. Esto es así porque las tecnologías de información y comunicación forman nuevos matices a la labor educativa y amplían el diapasón de información al alumno, razón por la cual se requiere de una preparación óptima del profesor en las herramientas y recursos TIC, así como una formación continua en función de las transformaciones que se suscitan en el proceso del docente universitario a nivel macro social para adaptarse paulatinamente a las condiciones actuales de clases virtuales.

Se puede decir entonces que, para dotar de competencias tecnológicas a los estudiantes no basta con introducir ordenadores y otras herramientas tecnológicas en el ámbito 
educativo universitario, es también imprescindible que los docentes estén preparados. Deben contar con la suficiente competencia en TIC, que les permita integrarlas en condiciones óptimas en los procesos de enseñanza y aprendizaje que se desarrollen en sus vídeo clases. Es preocupante el hecho de que, pese a la situación actual, frente a la pandemia que se vive, el docente de la vieja escuela aún no se tome en serio la responsabilidad de seguir educando, seguir enseñando de acuerdo a sus conocimientos y a otros nuevos, ya que el profesor debe estar actualizado en relación a las nuevas formas de enseñanza y temas que surgen con frecuencia en todos los ámbitos.

Como ya se ha planteado, esta realidad expuesta se presenta en el contexto universitario de la provincia de Huancayo (Perú), donde se puede apreciar que las prácticas educativas en las universidades no han logrado un gran propósito en los meses de aislamiento debido a la pandemia covid-19, ya que algunos docentes siguen siendo muy tradicionales en este caso y el cambio no es un hecho que se de en ellos, pues se basan en que el suministro de la información por parte del docente no debe darse a través de una pantalla de computador; sino utilizando como únicos recursos el pizarrón, cuaderno de apuntes, papelotes, entre otros; lo que convierte al estudiante en un ser pasivo, con poca habilidad para analizar y procesar información a través de otro recurso. Es conveniente hacer notar que si bien es cierto algunos docentes y estudiantes cuentan con los dispositivos tecnológicos apropiados, otros se encuentran en desventaja, ya que no tienen la misma suerte.

Agregando más elementos a este contexto problematizado en torno a lo que se vive en tiempos de pandemia respecto al docente y el uso de las herramientas tecnológicas para impartir sus clases, la realidad observada indica que los docentes asumen una actitud muy pasiva frente a los estudiantes, pues, sus acciones en el diario accionar no trascienden ni van más allá de una presentación en PowerPoint, una explicación sin el uso por lo menos de una pizarra digital interactiva, colocando a los estudiantes en un estatus metafórico de ser una cuenta bancaria donde lo ideal es depositar información para luego evaluarla de acuerdo a unos criterios que deberían estar por lo menos actualizados. Esto por supuesto, es un comportamiento intencional equivoco, siendo que, hoy más que nunca se ha transitado hacia la transformación educativa en todos los niveles, dejando claro que el desafío para el docente es avanzar e ir a la par de las transformaciones y los cambios tecnológicos, no rechazado las nuevas formas de enseñar y aprender sino más bien, aprovechando consonantemente sus beneficios.

También se considera parte de la realidad observada el hecho de que no puede negarse que, si hay un intento de uso de las tecnologías de información y comunicación por parte de los estudiantes y docentes, sin embargo, este uso es algo limitado por cuanto algunos no tienen acceso a internet y otros no dominan las herramientas tecnológicas. Y aquellos que pueden acceder de mejor forma y con señal, utilizan en el aprovechamiento de las redes sociales (Facebook, Hotmail, Gmail, entre otros); con fines personales, familiares, e individuales, pero pocas veces con fines educativos. 
La problemática descrita condujo a plantearse como interrogantes de interés para orientar la investigación, las siguientes preguntas:

- ¿Cuál es el nivel de conocimiento que tienen los docentes universitarios sobre las Tecnologías de la Información y la Comunicación en tiempos de pandemia?

- ¿Cuáles son los elementos de recursividad que brindan las tecnologías de la Información y la Comunicación a los docentes?

- ¿Cuáles son las estrategias de aprendizajes en las Tecnologías de la Información y la Comunicación como herramientas de enseñanza en tiempos de pandemia?

\section{OBJETIVOS DE LA INVESTIGACIÓN}

\section{Objetivo General}

Analizar el conocimiento sobre el uso de las Tecnologías de la Información y la Comunicación como herramienta de enseñanza de los docentes universitarios en tiempos de pandemia.

\section{Objetivos Específicos}

1. Identificar el nivel de conocimiento que tienen los docentes universitarios sobre las Tecnologías de la Información y la Comunicación en tiempos de pandemia.

2. Describir los elementos de recursividad que brindan las tecnologías de la Información y la Comunicación a los docentes.

3. Determinar las estrategias de aprendizajes en las Tecnologías de la Información y la Comunicación como herramientas de enseñanza en tiempos de pandemia.

\section{METODOLOGÍA}

Esta investigación se ubicó bajo una concepción paradigmática empírico analítico o positivista, con un enfoque cuantitativo, por tratarse de una realidad existente, palpable, observable y plenamente objetiva. En relación al tipo de investigación el presente estudio se encuentra sustentado en una investigación de campo de tipo descriptiva, la cual está enmarcada en analizar el uso de las Tecnologías de la Información y la Comunicación como herramienta de enseñanza de los docentes universitarios en tiempos de pandemia. El diseño de la investigación se ejecutó mediante un diseño de tipo transeccional, no experimental ya que los datos fueron recolectados directamente de la realidad objeto de estudio. La población del estudio estuvo constituida por 22 docentes. La muestra del estudio es censal, lo cual significa que la misma quedó conformada por el total de la población, es decir, 22 sujetos. 
En relación a las técnicas de recolección de datos, en esta investigación se utilizó la técnica de revisión documental de fuentes bibliográficas y electrónicas, cuyo procedimiento implico la revisión exhaustiva de la información, la selección de la misma y el correspondiente acopio documental. Cabe destacar que también se utilizó la observación directa dirigida a la población objeto de estudio, con la finalidad de recopilar toda la información necesaria para plantear y delimitar el problema, además conocer todos los pormenores de interés para esta investigación. Otra técnica que se empleó fue la encuesta la cual fue aplicada a los elementos de la muestra del presente estudio. Como instrumento se diseñó un cuestionario compuesto por 18 preguntas cerradas, de tipo dicotómicas, que permitió al consultado elegir una de dos alternativas: (a) Sí o (b) No.

Con relación a la validez de este estudio, se obtuvo mediante el juicio de expertos: un experto en diseño de instrumentos, que se aseguró que las preguntas estuviesen bien construidas desde el punto de vista sintáctico que no presentara ambigüedades o confusiones o ideas inconclusas en los ítems; un experto en metodología, quien revisó si las preguntas se adaptan tanto al diseño de la investigación como a los indicadores de las variables estudiadas (nivel de conocimiento sobre las TIC y herramienta de enseñanza) y a las técnicas que se utilizaron para procesar la información recogida por el instrumento; y un experto en contenido quien se aseguró que las preguntas se ajusten al marco teórico de la investigación y a la problemática estudiada así como también a los objetivos de la investigación.

Para obtener el coeficiente de confiabilidad de los instrumentos, se aplicó una prueba piloto a una población con las mismas características de la muestra de ésta investigación. Los resultados de dicha prueba suministraron los datos necesarios para determinar el índice de confiabilidad. Los resultados se procesaron matemáticamente mediante la técnica Kuder-Richardson (KR21) cuya fórmula es:

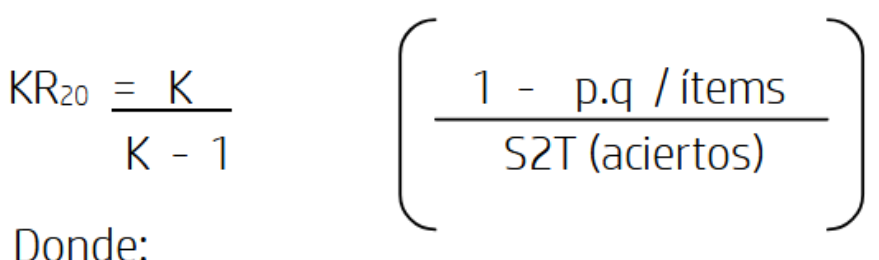

$$
\mathrm{KR}_{21}=\text { Técnica Kuder-Richardson Nivel de Confianza }=89 \%
$$

Después de ser aplicado el instrumento de recolección de datos, los investigadores procedieron a analizar el conjunto de datos obtenidos. Las técnicas de análisis de datos fueron las siguientes:

- Uso de la estadística descriptiva.

- Construcción de tablas de frecuencias de doble entrada. 
- Diagramación circular.

- Análisis e inferencia con apoyado en el sistema de autor fecha y la descripción e interpretación referente a los datos expuestos en cada diagrama.

\section{RESULTADOS}

Variable: nivel de conocimiento sobre las TIC.

\section{Conocimientos sobre las TICS:}

\begin{tabular}{|c|c|c|c|c|c|c|c|}
\hline \multirow{2}{*}{ N. I Item } & Descripción & \multicolumn{2}{|c|}{ SI } & \multicolumn{2}{|c|}{ NO } & \multicolumn{2}{c|}{ TOTAL } \\
\hline \multirow{2}{*}{1} & $\begin{array}{c}\text { ¿Damina eficientemente los programas } \\
\text { básicos de computación Word, Excel, } \\
\text { PowerPoint? }\end{array}$ & 10 & 45,45 & 12 & 54.55 & 22 & 100 \\
\hline
\end{tabular}

Cuadro 1. Fuente: Base de datos de la investigación (2020)

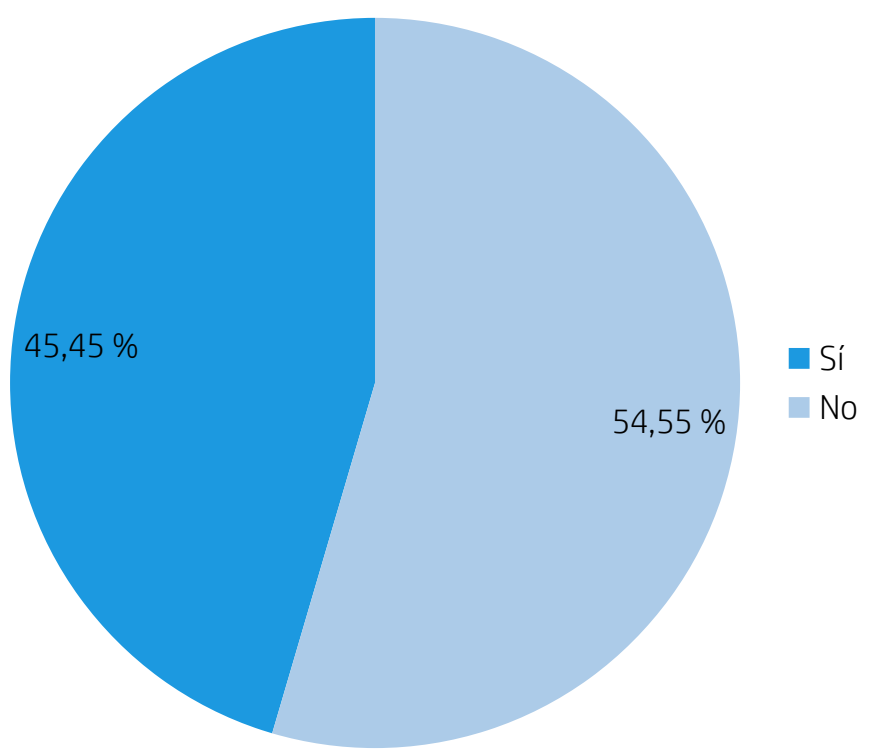

Gráfico 1: Distribución porcentual del indicador conocimiento sobre las TIC.

\section{Análisis}

La postura del 45,45\% de los encuestados coincide en que dominan eficientemente los programas básicos de computación Word, Excel, Powert Point, mientras que el 54,55\% restante afirma no posee dicho dominio, situación que es severamente preocupante ya que en la actualidad debido a la situación de aislamiento por efectos de la pandemia por 
covid-19 se vuelve un requisito indispensable para la presentación y explicación de las clases a través de las vídeo clases.

Habilidades en el manejo de programas y herramientas virtuales para vídeo clases:

\begin{tabular}{|c|c|c|c|c|c|c|c|}
\hline \multirow{2}{*}{ N.. Item } & Descripción & \multicolumn{2}{|c|}{ SI } & \multicolumn{2}{|c|}{ NO } & \multicolumn{2}{c|}{ TOTAL } \\
\hline 4 & $\begin{array}{c}\text { fa } \\
\text { involucra en uso programas y herra- } \\
\text { mientas virtuales en el desarrollo de sus } \\
\text { clases? }\end{array}$ & 13 & 59,09 & 9 & 40,91 & 22 & 100 \\
\hline
\end{tabular}

Cuadro 2. Fuente: Base de datos de la investigación (2020)

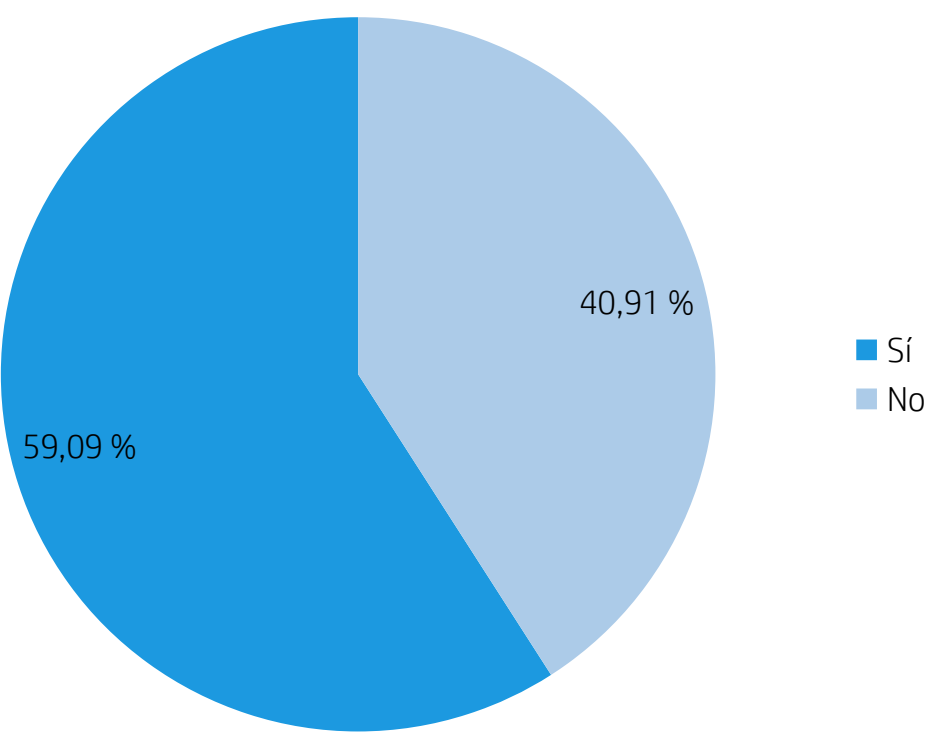

Gráfico 2: Distribución porcentual del indicador habilidades en el manejo de programas y herramientas virtuales.

\section{Análisis}

La toma muestral consultada permitió evidenciar que en el 59,09\% de los casos, el docente involucra en el manejo programas y herramientas virtuales en el desarrollo de sus clases, mientras que el 40,91\% restante no lo hace. De esta aseveración se infiere que el docente no está formado y/o preparado para enfrentar el reto de las clases no presenciales por la costumbre de la presencialidad que desde siempre se había mantenido. 


\section{Dificultad para manejar internet:}

\begin{tabular}{|c|c|c|c|c|c|c|c|}
\hline \multirow{2}{*}{ N.. Item } & Descripción & \multicolumn{2}{|c|}{ SI } & \multicolumn{2}{c|}{ NO } & \multicolumn{2}{c|}{ TOTAL } \\
\cline { 3 - 8 } & fa & $\%$ & fa & $\%$ & fa & $\%$ \\
\hline 7 & $\begin{array}{c}\text { impartir sus clases haciendo uso del } \\
\text { Internet es un reto que usted se había } \\
\text { planteado? }\end{array}$ & 3 & 14 & 19 & 86 & 22 & 100 \\
\hline
\end{tabular}

Cuadro 3. Fuente: Base de datos de la investigación (2020)

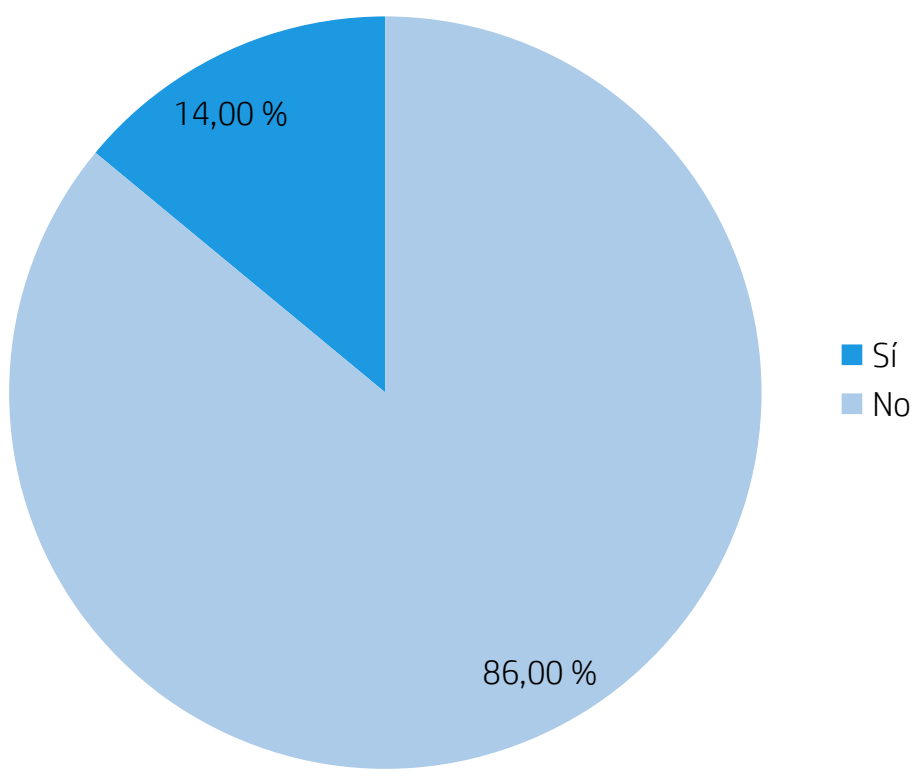

Gráfico 3: Distribución porcentual del indicador dificultad para manejar la internet.

\section{Análisis}

Los porcentajes expuestos reflejan que un $86 \%$ de los docentes que no se habían planteado usar el internet. Este planteamiento es expresado por Islas (2007), cuando enfatiza el docente debe usar la internet para disminuir la monotonía en el aula. El 14\% de la toma muestral restante considera que usar el internet no es un reto para ellos, pues, asumen como mecanismo adecuado para sus clases. Con ello se pone en evidencia la marcada tendencia de seguir propiciando estrategias de enseñanza y aprendizajes tradicionales, que no son cónsonas a los avances tecnológicos y a la forma de trabajo remoto que se está llevando a cabo con los estudiantes en la actualidad a causa del aislamiento social. La consecuencia directa de ello es que la actitud docente no se nota creativa y no se encuentra formado para asumir retos tan importantes como los de aplicar nuevas estrategias y herramientas para poder mantener al estudiante motivado en las clases virtuales. 


\section{Percepción de las TIC por parte de los estudiantes:}

\begin{tabular}{|c|c|c|c|c|c|c|c|}
\hline \multirow{2}{*}{ N.. Item } & Descripción & \multicolumn{2}{|c|}{ SI } & \multicolumn{2}{|c|}{ NO } & \multicolumn{2}{c|}{ TOTAL } \\
\cline { 3 - 8 } & fa & $\%$ & fa & $\%$ & fa & $\%$ \\
\hline 10 & $\begin{array}{c}\text { ¿El uso de Internet estimula la atención } \\
\text { de los estudiantes? }\end{array}$ & 14 & 63,64 & 8 & 36,36 & 22 & 100 \\
\hline
\end{tabular}

Cuadro 4. Fuente: Base de datos de la investigación (2020)



Gráfico 4: Distribución porcentual del indicador dificultad para manejar la internet.

\section{Análisis}

El 68\% de los docentes señalan que el uso de internet motiva a sus estudiantes. Esta evidencia es un reforzador de la importancia del buen uso del internet por parte del docente que debe actualizar sus estrategias para mejorar la calidad del proceso de enseñanza y estar a la vanguardia de la situación actual, en donde la creatividad e innovación por parte del docente es esencial para impartir sus clases a través de un aula virtual que puede ser síncrona o asíncrona. Solo el 36,36\% restante señala que sienten que sus estudiantes no se encuentran motivados por las clases a través de las tecnologías basadas en el internet. En tanto, se infiere que el docente, debe prepararse y formarse para los cambios que se han suscitado en estos tiempos de pandemia, por lo que las capacitaciones podrían ser una buena opción que las universidades en todo caso deberían asumir y tocar temas de relevancia en estrategias virtuales sobre metodologías de aula invertida, metodología basa en caso, en proyecto. Asimismo, motivarlos a que utilicen programas que capten la atención de sus estudiantes. 
Uso didáctico de las TIC:

\begin{tabular}{|c|c|c|c|c|c|c|c|}
\hline \multirow{2}{*}{ N.. Item } & Descripción & \multicolumn{2}{|c|}{ SI } & \multicolumn{2}{c|}{ NO } & \multicolumn{2}{c|}{ TOTAL } \\
\hline \multirow{2}{*}{15} & $\begin{array}{c}\text { ¿Considera usted que mediante el uso } \\
\text { de internet y las diferentes herramien- } \\
\text { tas virtuales se estimula el trabajo } \\
\text { cooperativo de los estudiantes? }\end{array}$ & 18 & 81,82 & 4 & 18,18 & 22 & 100 \\
\hline
\end{tabular}

Cuadro 5. Fuente: Base de datos de la investigación (2020)

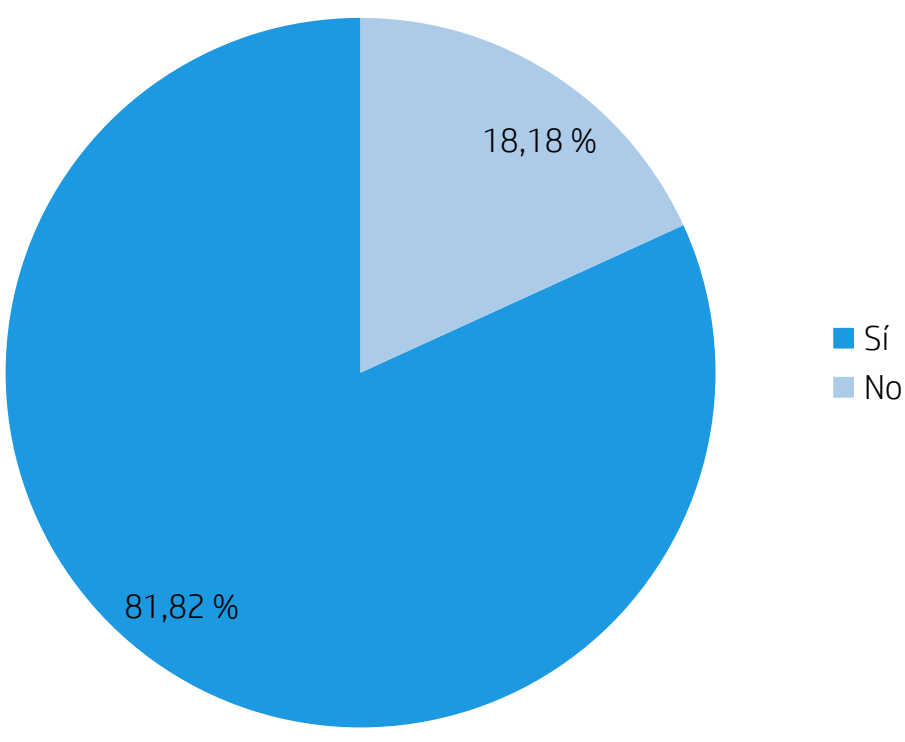

Gráfico 5: Distribución porcentual del indicador dificultad para manejar la internet.

\section{Análisis}

La opinión de los encuestados permite observar que en un (86\%) están de acuerdo en afirmar que mediante el uso del internet se estimula el trabajo cooperativo de los estudiantes, situación que es muy positiva en la actualidad. Al respecto, el 14\% restante opina de manera contraria. En este sentido, la interpretación apunta a una crítica reflexiva debido a que el uso de las tecnologías desde la visión del docente o del estudiante, tiende más bien a reducir el proceso de comunicación e interacción humana, de allí que el trabajo en equipo se sustituye más bien por el trabajo individual.

\section{DISCUSIÓN}

Las TIC son herramientas esenciales de trabajo y aprendizaje en la sociedad del conocimiento donde la generación, procesamiento y transmisión de información es un factor 
esencial de poder y productividad, en consecuencia, resulta cada vez más necesario educar para la sociedad de la información desde las etapas más tempranas de la vida escolar, con el fin de que el individuo pueda llegar al entorno universitario con el conocimiento y competencias necesarias para asumir los retos de la virtualidad en la que se vive actualmente. Para que pueda haber un verdadero impacto de las TIC en la configuración de nuevos modos de enseñanza y aprendizaje se requiere de una visión integradora y creativa del docente, en donde exista interés por prepararse y estar a la vanguardia de los cambios que subyacen día a día.

De acuerdo a la encuesta aplicada se pudo evidenciar que la mayoría de los docentes no se encuentran preparados para asumir con creatividad e innovación las clases de manera remota o clases virtuales. Asimismo, muchos no motivan a sus estudiantes con las diversas formas, herramientas, programas y planificación necesaria para captar la atención del estudiante, lo que requiere una puesta en práctica de aplicación de herramientas y recursos que ofrece la internet. El docente está llamado a investigar y estar en correspondencia con lo que se vive en la actualidad en donde todo es asumido de manera virtual. Muchos programas que existen actualmente le dan tanto al docente como al estudiante la posibilidad de reinventarse frente a esta crisis social y sanitaria que está pasando a nivel mundial.

Un ejemplo de la innovación es la plataforma de Google, la cual ofrece diversas herramientas que se encuentran en el site, desde donde el docente puede hacer uso de ellas y de forma gratuita. La gran plataforma posee desde pizarras interactivas, formularios para exámenes o diagnósticos, aula virtual o salón de clases (classroom), calendario, comunicación por Meet o Hangouts, Drive (para guardar información en la nube y realizar trabajo colaborativo de forma simultánea), Blogger (para presentar la información al público) y documentos, entre otros. Con esta cantidad de herramientas y que solo es de una plataforma que es Google, el docente puede manejar de forma virtual sus clases de manera síncrona y asíncrona.

De igual forma se presenta una diversidad de recursos en internet que pueden ser usados por docentes y estudiantes de manera tal que se logre optimizar tiempo y calidad educativa a través de una plataforma. Tal es el caso de plataformas como GoConqr, Edpuzzle que es una aplicación gratis y con fines educativos que permite la creación de cuestionarios de evaluación. Y así como las anteriores hay variedad que solo se tienen que buscar en internet. En el caso de las presentaciones online se tiene desde el tan usado PowerPoint hasta el Prezzi, Emaze y otros que permiten captar la atención con presentaciones dinámicas.

Por otro lado, es importante también reconocer, que el ordenador y las nuevas tecnologías son de gran ayuda al docente, cuestión que es coincidente con las observaciones sostenidas por (Quines, 2009), al señalar que las nuevas tecnologías son un gran elemento (denominador) de ayuda al docente. En ese sentido, los encuestados aseveraron la preeminencia del uso de los recursos tecnológicos en la actualidad, sin embargo, dicha 
aseveración solo es teórica, pues, la realidad empírica evidencia que en la práctica docente no están debidamente preparado para tal fin y lo hacen solo por cumplir.

Existe una marcada tendencia de seguir propiciando estrategias de enseñanza y aprendizajes tradicionales, que no son cónsonas a los avances tecnológicos de la actualidad y con lo que se vive producto del aislamiento social.

\section{CONCLUSIONES}

Se concluye de acuerdo a la información obtenida a través de las encuestas y el desarrollo de la investigación como tal, que la postura de un 45,45\% de los encuestados coincide en que dominan eficientemente los programas básicos de computación Word, Excel, PowerPoint, mientras que el 54,55\% restante afirma no posee dicho dominio, situación que es severamente preocupante ya que en la actualidad debido a la situación de aislamiento por efectos de la pandemia por covid-19 se vuelve un requisito indispensable para la presentación y explicación de las clases a través de las vídeo clases.

De la misma forma, se evidencio que existe un marcado desinterés sobre los beneficios que ofrecen las TIC a la praxis educativa, situación que se puede corregir con la constante formación docente sobre las bases del auge virtual en tiempos de pandemia. El docente no está formado y/o preparado para enfrentar el reto de las clases no presenciales por la costumbre de la presencialidad que desde siempre se había mantenido. Y, existe una marcada tendencia de seguir propiciando estrategias de enseñanza y aprendizajes tradicionales, que no son cónsonas a los avances tecnológicos y a la forma de trabajo remoto que se está llevando a cabo con los estudiantes en la actualidad a causa del aislamiento social. La consecuencia directa de ello es que la actitud docente no se nota creativa y no se encuentra formado para asumir retos tan importantes como los de aplicar nuevas estrategias y herramientas para poder mantener al estudiante motivado en las clases virtuales.

El docente debe prepararse y formarse para los cambios que se han suscitado en estos tiempos de pandemia, por lo que las capacitaciones podrían ser una buena opción que las universidades en todo caso deberían asumir y tocar temas de relevancia en estrategias virtuales sobre metodologías de aula invertida, metodología basa en caso, en proyecto. Asimismo, motivarlos a que utilicen programas que capten la atención de sus estudiantes. El uso de las tecnologías desde la visión del docente o del estudiante, tiende más bien a reducir el proceso de comunicación e interacción humana, de allí que el trabajo en equipo se sustituye más bien por el trabajo individual.

Un docente actualizado, formado en el uso de las tecnologías y con una actitud amigable y no resistente a los avances tecnológicos, tendrá una mayor participación en los escenarios de aprendizajes considerando que su acervo está más nutrido que nunca y que es necesario para proyectar esta virtualidad que día a día crece aún más. Se considera que el uso de las tecnologías de información y comunicación incorporadas a la práctica 
docente, permite una enseñanza y aprendizaje más adecuado que nunca a la sociedad del conocimiento en el que se vive en la actualidad, donde los ordenadores, las redes sociales, la información virtual es un común denominador en la vida del ser humano.

Los docentes consideran que las TIC aumentan la responsabilidad del estudiante en el propio proceso de aprendizaje. Tal responsabilidad es un desafío hoy día para los docentes, ya que no solo deben vigilar que tal herramienta se emplee adecuadamente, sino que también, deben crear conciencia en los estudiantes para que sean prudentes a apoyarse en las TIC sin que se lleguen a generar los vicios mal entendidos que normalmente se disfrazan de facilismo tales como: El corta y pega, el desinterés por la lectura crítica y reflexiva de los libros, el uso de los dispositivos móviles solo para la interacción en redes sociales (tomando en cuenta que a través de estas redes también se puede ganar en la obtención de información de interés, ya que se promocionan cursos, talleres gratuitos), entre otros.

\section{REFERENCIAS}

ÁVILA, T. (2011). Alfabetizaciones y tecnologías de la información y la comunicación. Madrid: Síntesis.

DÍAZ-BARRIGA, A. (2013). Tic en el trabajo del aula. Impacto en la planeación didáctica. Revista Iberoamericana de educación superior, 3-21. Disponible en: [Enlace]

QUINES, R. (2009). Las nuevas tecnologías como elemento dinamizador del aprendizaje significativo en el contexto de la educación a distancia. Repositorio de la Universidad Rómulo Gallegos.

RICHTA, E. (2010). Cómo valorar la calidad de la enseñanza basada en las TIC. Pautas e instrumentos de análisis. Barcelona: Graó.

URRIBARRÍ, R. (2002). Educación y TIC: Nuevas prácticas pedagógicas. Laboratorio de Investigación Educativa, 1-10.

VELASCO, O. y. (2009). Filosofía de la educación. Madrid: Síntesis. 